\title{
Conversion of a chirped Gaussian pulse to a soliton or a bound multisoliton state in quasi- lossless and lossy optical fiber spans
}

\author{
Jaroslaw E. Prilepsky \\ B. Verkin Institute For Low Temperature Physics and Engineering, 61103 Kharkov, Lenin Avenue 47, Ukraine
}

Stanislav A. Derevyanko* and Sergei K. Turitsyn

Photonics Research Group, Aston University, B4 7ET Birmingham, UK

Received December 11, 2006; revised February 7, 2007; accepted February 11, 2007; posted March 8, 2007 (Doc. ID 77941); published May 17, 2007

\begin{abstract}
The formation of single-soliton or bound-multisoliton states from a single linearly chirped Gaussian pulse in quasi-lossless and lossy fiber spans is examined. The conversion of an input-chirped pulse into soliton states is carried out by virtue of the so-called direct Zakharov-Shabat spectral problem, the solution of which allows one to single out the radiative (dispersive) and soliton constituents of the beam and determine the parameters of the emerging bound state(s). We describe here how the emerging pulse characteristics (the number of bound solitons, the relative soliton power) depend on the input pulse chirp and amplitude. () 2007 Optical Society of America

OCIS codes: $060.5530,190.5530$.
\end{abstract}

\section{INTRODUCTION}

Integrable nonlinear systems have a special role in modern mathematics, physics, and various practical applications. In the context of nonlinear optics, the nonlinear Schrödinger equation (NLSE) [which is an example of a widely applicable and thoroughly studied integrable nonlinear model solved by the inverse scattering transform $\operatorname{method}^{1}$ (IST)] has played an important part in modeling signal propagation in single-mode optical fibers. ${ }^{2-5}$ Since the NLSE possesses stable soliton solutions, it was proposed to use soliton pulses for optical data transmission and storage (see, e.g., Refs. 2, 4-7 and references therein).

Some recent examples of applications of optical solitons in these areas include all-optical regeneration using conversion of return-to-zero (RZ) signals into conventional solitons $^{8,9}$ and all-optical picosecond-pulse packet buffers based on four-wave-mixing loading and intracavity soliton control. ${ }^{10}$ It was shown in Refs. 8 and 9 that the efficiency of carrier signal control can be enhanced by periodic conversion of an RZ signal into a classical soliton. The benefit of RZ signals [e.g., dispersion-managed (DM) solitons] to fundamental soliton conversion is that the stabilization of conventional solitons is more efficient compared with an arbitrary RZ pulse. The practical use of true NLSE solitons, however, requires that fiber loss is compensated exactly along the fiber span by distributed amplification.

Recently, such a quasi-lossless fiber span has been designed $^{11,12}$ and experimentally implemented ${ }^{11,13}$ using an amplifier scheme that combines second-order bidirectional pumping and fiber Bragg grating reflectors at the secondary pump wavelength, which effectively converts a transmission span into an ultralong laser. It has been demonstrated experimentally ${ }^{11,13}$ that this scheme allows one to effectively achieve quasi-lossless transmission over span lengths up to $75 \mathrm{~km}$ with virtually zero signal power variation. This result has paved the way for the practical implementation of integrable nonlinear systems in optical fiber devices. In practical terms, the quasi-lossless regime is modeled here by the ideal lossless NLSE, so in what follows, we will not distinguish between the two.

In this paper, we revisit the problem of pulse-to-soliton conversion in both lossless and lossy fibers. We consider conversion of a linearly chirped Gaussian pulse-the waveform, which is especially important for a variety of fiber-optics applications. For example, in the optical regeneration technique, a standard chirped-RZ signal (well approximated by chirped Gaussian pulses) is converted into the NLSE soliton for efficient filtering and then launched again into the fiber line. ${ }^{8,9}$ Also it is a known fact among the experimentalists that it is quite difficult to control the chirp for sech-shaped input pulses. Therefore if one tries to produce supersoliton inputs $N$ sech $t$ with large $N$, the chirp becomes uncontrollable and difficult to quantify. On the other hand, Gaussian pulses can be easily produced and controlled, which makes them perfect candidates for the soliton sources. Another important reason why we picked Gaussian pulse shapes in the first place is that the shape of a DM soliton is often quite close to Gaussian. Therefore if one wishes to use the quasilossless loop as a storage facility for DM solitons, it is crucial to investigate how the DM pulse shape transforms into the integrable nonlinear Schrödinger solution and back. 
It is worthwhile now to clarify some terminology. Historically, in the context of the NLSE, the term "bound states" (breathers) was introduced in the original paper by Zakharov and Shabat. ${ }^{1}$ It has become a common term ever since and is widely used throughout the literature dedicated to the NLSE. In IST, the term bound states is generally understood in a sense that the individual solitons in the pulse do not disperse and remain localized (bound). It is more typical, however, in nonlinear science, to use this term for structures having nonzero binding energy. In this paper, we will nevertheless stick to the first definition and will use the term "bound-multisoliton state," although these structures technically have zero binding energy.

Note that the problem of conversion of the pulses having different shapes into NLSE solitons has already been studied in various contexts, both analytically and numerically, ${ }^{14-28}$ including the study of chirped Gaussiantype input pulses. Let us recall some of these results, in order to clarify what is new in our studies. The authors of Ref. 14 were the first who addressed the problem of the formation of multisoliton states emerging from chirped input pulses in the NLSE. Using the numerical solutions of the associated direct Zakharov-Shabat scattering problem $^{1}$ (ZSSP) attached to the NLSE (the input pulse profiles play the role of "potentials" for the associated ZSSP), they inferred that the area theorem giving the minimal value of the area of the monotonic unchirped input profile sufficient to create a soliton state, was no longer valid for the chirped profiles. The authors of Ref. 14 also studied the multisoliton conversion of the chirped Gaussian input profile but, however, the analysis was limited only to certain values of the chirp parameter. Later, in Ref. 15, the author studied the generation of bright spatial soliton beams from a linearly chirped Gaussian input waveform (the direct form of the input pulse was also somewhat different from that considered in the present paper) and compared the results with those for sechshaped input profiles to demonstrate the distinctions. The results obtained apply to a single-soliton regime, and an approximate analytical formula for the amplitude of the emerging soliton was derived in the case of unchirped pulses. Burak ${ }^{16}$ dealt with a similar problem, but for real, symmetric, and spatially modulated Gaussian pulses. In Ref. 17, the authors demonstrated both numerically and experimentally, the breakup of a strongly prechirped sech pulse into a train of fundamental solitons in lossless lowdispersion optical fibers. A similar effect was considered analytically in Refs. 18 and 19, while in Ref. 20, the stability of linearly chirped sech pulses was demonstrated numerically, In Refs. 21 and 22, a variational approach was used to approximate the eigenfunctions (the so-called Jost functions) of the ZSSP associated with the different initial pulse shapes of the NLSE. This approach allowed the approximate values for discrete ZSSP eigenvalues to be obtained, which correspond to the bound multisoliton states, as a function of the input shape parameters. However, in Ref. 29, it was pointed out that the results of variational approaches can sometimes fail to correctly describe the spectrum of the ZSSP if the chosen ansatz is not flexible enough; one example of such a case is the interaction of solitons with radiation. In the latter case, a special type of ansatz is required (see Ref. 30). In Ref. 23, a WKB approach was applied to the problem of soliton formation from a variety of different shapes for the initial pulses, while in Ref. 25, a combination of variational and WKB methods was used to find the decomposition of the initial pulses into multisoliton states.

However most of the works cited apply to the singlesoliton regime and give only approximate values of the ZSSP eigenvalues; in the current paper, we generally deal with a more complicated case when one needs to study multisoliton pulse decomposition. Moreover when concerned with the lossy case, no analytical solution for the associated ZSSP is available at all, so we will resort to the numerical solution of the associated ZSSPs.

To conclude the introductory part on multisoliton conversion, it is worthwhile to mention that the integrability of a system is not a necessary condition to attain stable pulse propagation. One such example is provided by the solitons in the DM systems mentioned earlier. A recent paper $^{31}$ proposes a new method of analyzing the soliton content of the pulse, and the method does not rely on the property of system integrability (and hence it does not incorporate the considerations based on the solution of the associated direct-scattering problem), and it may be applied to nonintegrable systems such as DM transmission lines.

In the present paper, we study soliton conversion in a fiber span both in the lossy and the quasi-lossless regimes. The numerical values of loss we use in our simulations actually imply that the system is far from integrable. In this case, we use a combination of a split-step Fourier method to propagate the pulse in a lossy fiber and ZSSP analysis to study its spectral content at each point in space. When solving the direct ZSSP numerically, we use a well-documented piecewise constant approximation for the potential (see, e.g., Ref. 26 for the detailed description). Though for the systems far from integrable, the decomposition into soliton "modes" via ZSSP itself is not very meaningful; its application makes perfect sense when one considers a span of lossy fiber immediately followed by a span of quasi-lossless fiber. Then the ZSSP spectral content at the output of the lossy span determines completely the further integrable evolution of the pulse. We will examine the conversion of the inputchirped Gaussian pulses and determine how the characteristics of the emerging soliton states, such as the number of the bound states, the relative soliton power, and Hamiltonian, depend on the amplitude and chirp of the input Gaussian pulse in both quasi-lossless and lossy regimes. Note that previously reported results on this problem $^{15}$ were concerned with a single-soliton regime and lossless case only.

This paper is organized as follows. In Section 2, we describe the normalization of the NLSE in an optical fiber, introduce basic scales, and describe the multisoliton decomposition addressed in the current paper. In Section 3, we present results of numerical multisoliton decomposition of the chirped Gaussian pulse in the quasi-lossless 
regime. Section 4 presents a similar analysis for a $25 \mathrm{~km}$ lossy fiber span.

\section{MODEL}

The evolution of optical pulses along the fiber span is governed by the $\mathrm{NLSE}^{3}$ :

$$
i \psi_{z}+\frac{i \alpha}{2} \psi-\frac{\beta_{2}}{2} \psi_{t t}+\gamma|\psi|^{2} \psi=0 .
$$

This equation describes the propagation of a light envelope $\psi(z, t)$ in a lossy optical fiber with constant groupvelocity dispersion. Here $\alpha=\tilde{\alpha} \times 0.1 \ln 10$ is the fiber power loss (recalculated from the corresponding quantity $\widetilde{\alpha}$ measured in $\mathrm{dB} / \mathrm{km}), \beta_{2}$ is the second-order dispersion coefficient (measured in $\mathrm{ps}^{2} / \mathrm{km}$ ), and $\gamma$ stands for the nonlinear coefficient (measured in $\mathrm{W}^{-1} \mathrm{~km}^{-1}$ ). It is customary to operate with Eq. (1) in dimensionless soliton units. More specifically, if we introduce the pulse width $T_{0}$, the so-called dispersion length $L_{D}=T_{0}^{2} /\left|\beta_{2}\right|$, and the characteristic power $P_{0}=\left(\gamma L_{D}\right)^{-1}$, we can rewrite Eq. (1) in dimensionless rescaled units $t \rightarrow t / T_{0}, z \rightarrow z / L_{D}, \psi$ $\rightarrow \psi / \sqrt{P_{0}}, \alpha \rightarrow \alpha L_{D}$, as

$$
i \psi_{z}+\frac{i \alpha}{2} \psi \mp \frac{1}{2} \psi_{t t}+|\psi|^{2} \psi=0
$$

The sign of the third term in Eq. (2) is determined by the sign of the dispersion coefficient $\beta_{2}$ in Eq. (1). In what follows, we will always assume constant anomalous dispersion $\left(\beta_{2}<0\right)$, which corresponds to the "+" sign in Eq. (2). We will now use the dimensionless notation and will return to the real world units only when a quantitative description is desirable. For the specific values of parameters, we use standard single-mode telecommunication fibers with $\beta_{2}=20 \mathrm{ps}^{2} / \mathrm{km}, \widetilde{\alpha}=0.2 \mathrm{~dB} / \mathrm{km}$, and $\gamma$ $=3 \mathrm{~W}^{-1} \mathrm{~km}^{-1}$. We will consider pulses of width $T_{0}=22 \mathrm{ps}$, which yield $L_{D}=25 \mathrm{~km}$ and $P_{0}=13 \mathrm{~mW}$. The dimensionless loss is then computed as $\alpha=1.15$. In the quasi-lossless regime, of course, losses are neglected, and so in that case, one deals with the integrable version of the NLSE with $\alpha=0$ in Eq. (2). In the absence of loss, it is known that Eq. (2) possesses a family of soliton solutions. Let us write down, for reference, the general form of a singlesoliton solution in the lossless case:

$$
\psi(z, t)=\frac{2 \eta}{\cosh \left[2 \eta\left(t-T_{0}+2 \xi z\right)\right]} e^{-2 i \xi t+i \sigma_{0}+2\left(\eta^{2}-\xi^{2}\right) z} .
$$

This anzatz defines four basic parameters of a single soliton: amplitude $2 \eta$, frequency (or velocity in time domain) $2 \xi$, initial position $T_{0}$, and initial phase $\sigma_{0}$.

More generally in the absence of loss, the fundamental understanding of the pulse behavior can be provided by decomposing the wave field into "normal" coordinates, i.e., the soliton and nonsoliton (quasi-linear radiation) components, also termed as the nonlinear spectral data. Such a decomposition of an arbitrary profile implies the solution of the associated direct scattering problem, i.e., $\mathrm{ZSSP}^{1}$ :

$$
\frac{\mathrm{d} \varphi_{1}}{\mathrm{~d} t}=\psi \varphi_{2}-i \zeta \varphi_{1}
$$

$$
\frac{\mathrm{d} \varphi_{2}}{\mathrm{~d} t}=-\psi^{*} \varphi_{1}+i \zeta \varphi_{2}
$$

Generally, an initial condition $\psi(0, t)$ gives rise to a continuous real spectrum of eigenvalues $\zeta$ (associated with the background radiation), and in addition to the continuous spectrum, the ZSSP can also support a discrete spectrum - the finite number of complex-valued eigenvalues $\zeta_{k}=\xi_{k}+i \eta_{k}$ located in the upper complex half-plane of $\zeta$. The discrete part of spectrum is associated with the soliton part of the solution and for each $k$, the real part of the complex eigenvalue, $\xi_{k}$, is half the corresponding soliton frequency, and the imaginary part $\eta_{k}$ is half the soliton amplitude. The key to the IST technique is the fact that the discrete eigenvalues (soliton parameters) are integrals of motion, which makes the evolution of spectral data with propagation distance, $z$, trivial. Another important property of solutions is that the radiative part generally disperses away from the soliton solution after a sufficient propagation distance. ${ }^{29}$ Therefore, an arbitrary localized input waveform $\psi(0, t)$ asymptotically evolves into a multisoliton bound state with negligibly small corrections due to radiative part (provided that there exists at least one discrete eigenvalue of the ZSSP associated with the input profile).

The decomposition in the lossless case can be performed directly by inserting the chirped Gaussian pulse into a direct spectral transform solver. This gives us the dependence of the eigenvalues and, therefore, the asymptotic multisoliton bound-state characteristics, on the input pulse parameters. It is appropriate to note that the analytical solution of the ZSSP is known just for a limited number of input waveforms, ${ }^{32}$ and for the chirped profiles, one usually has to resort to approximate solutions or numerical techniques. ${ }^{14,15}$ When dealing with lossy propagation, we carry out the numerical integration of the lossy NLSE (2) with a chirped Gaussian pulse as an input profile and then take the output profiles as the ZSSP potentials for subsequent analysis. Eventually the final soliton-state characteristics are again analyzed as functions of the input shape amplitude and chirp values.

The lossless NLSE possesses an infinite number of conserved quantities (integrals of motion) that can be expressed in terms of scattering data. We will consider here the following integrals of motion for the NLSE, which are often taken as the fundamental characteristics of the solution: total pulse power, often referred to as the number of bound quasi-particles, $\mathcal{N}$ :

$$
\mathcal{N}=\int_{-\infty}^{\infty} \mathrm{d} t|\psi|^{2}
$$

and Hamiltonian $\mathcal{H}$ :

$$
\mathcal{H}=\frac{1}{2} \int_{-\infty}^{\infty} \mathrm{d} t\left(\left|\psi_{t}\right|^{2}-|\psi|^{4}\right)
$$

Both integrals of motion can be expressed through the scattering data with the obvious decomposition into the soliton and radiation contributions: 


$$
\begin{gathered}
\mathcal{N}=4 \sum_{k} \eta_{k}+\int_{-\infty}^{\infty} \mathrm{d} \zeta n(\zeta), \\
\mathcal{H}=16 \sum_{k}\left(\xi_{k}^{2} \eta_{k}-\eta_{k}^{3} / 3\right)+\int_{-\infty}^{\infty} \mathrm{d} \zeta h(\zeta),
\end{gathered}
$$

Total power and Hamiltonian are important quantities because in the lossless case, being integrals of motion, they provide valuable information about the structure of the solution. In the fiber-optical applications, one is interested in the amount of power actually contained in the soliton part of a solution and how it compares with the total power of the solution.

\section{MULTISOLITON DECOMPOSITION OF A CHIRPED GAUSSIAN}

Consider a linearly chirped Gaussian initial pulse having the form

$$
\psi^{0}(t, 0)=A \exp \left[-\frac{1}{2}(1+i C) t^{2}\right],
$$

where $A$ is the pulse amplitude and $C$ is the chirp parameter. Our goal is to carry out numerical spectral analysis of the ZSSP (4) with the potential Eq. (9) inserted. The following properties of ZSSP associated with the chirped Gaussian profile are useful here ${ }^{14,15}$ : (i) the scattering data are invariant under the change of the chirp sign, $C$ $\rightarrow-C$; (ii) the discrete eigenvalues for the chirped symmetric Gaussian profile Eq. (9) lie on the upper part of the imaginary half-axis of the $\zeta$ plane.

The numerical method for the solution of ZSSP, Eq. (4), utilizes the piecewise constant approximation of the input profile $^{26}$ used as a potential of the associated ZSSP. The sensitivity of the method (i.e., the lower threshold of the absolute values for the eigenvalues above which we were able to recognize the presence of a new soliton) was $\left|\zeta_{k}\right|$ $\sim 10^{-3}$.

The results of our analysis are given in Figs. 1-3. If one increases the input profile amplitude, the number of solitons evidently becomes bigger. However the increase of the chirp value brings about the opposite effect tending to diminish the number of solitons and also the quantities of the power transferred into the soliton part as well as the amount of the Hamiltonian associated with the solitons.

To illustrate the effect of the chirp on the number of created solitons in Figs. 4(a)-4(c), we show the propagation of the chirped Gaussian pulse in lossless optical fibers $[\alpha=0$ in Eq. (2)] for three different sets of input parameters. The following results were obtained by solving the NLSE (2) with initial profile Eq. (9) using the secondorder split-step Fourier method. ${ }^{3}$ The first graph [Fig. 4(a)] corresponds to $A=2, C=0$, and the results of our simulation demonstrate the creation of the two-soliton bound state. The next one [Fig. 4(b)] corresponds to the same amplitude, but the value of the chirp $(C=2)$ places the system in the single-soliton regime [see the density plot in Fig. 4(b)]. Spatial oscillations of the amplitude decay slowly, approaching the predicted single-soliton amplitude $A_{\text {sol }} \approx 2.37$. Finally, Fig. 4(c) corresponds to $A$
$=2, C=7$. In this case, one can observe that, in accordance with Fig. 4(c), no solitons were created during the propagation. It is also instructive to plot explicitly the amplitude threshold for the creation of 1,2 , 3 soliton states versus the value of chirp as done in Fig. 5 .

It is useful to point out the difference between the linear and nonlinear chirp where the phase variation decays at the wings of the pulse. This difference was mentioned in the paper by Desaix et al. ${ }^{19}$ For the linear chirp, the number of soliton states and net soliton power decrease monotonically with the chirp for the sech-shaped pulses, which is in agreement with our results for the linearly

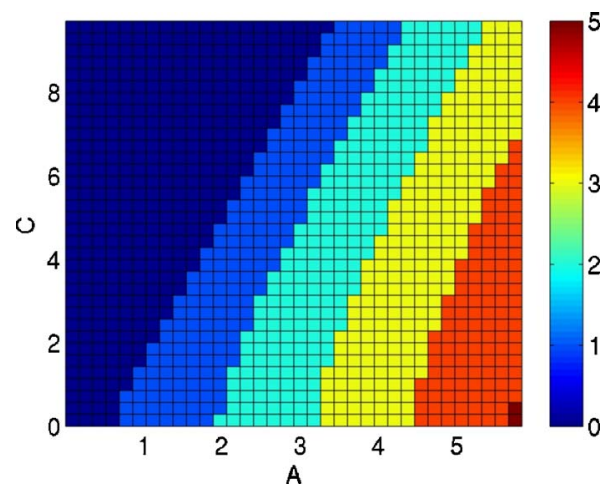

Fig. 1. (Color online) Density plot of the number of solitons [numbers of discrete eigenvalues for the associated ZSSP, Eq. (4)] generated from the input-chirped Gaussian profile, Eq. (9), as a function of the amplitude $A$ and chirp $C$ values. Lossless case.

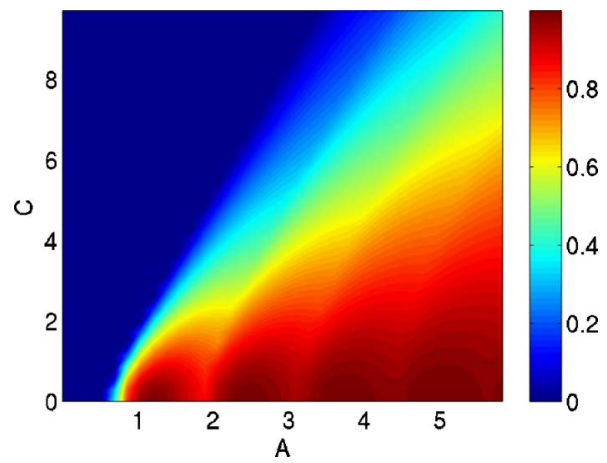

Fig. 2. (Color online) Density plot of the relative power of the solution, $\mathcal{N}_{\text {soliton }} /\left(\mathcal{N}_{\text {radiation }}+\mathcal{N}_{\text {soliton }}\right)$, see Eq. (7), generated from the input-chirped Gaussian profile, Eq. (9), as a function of the amplitude $A$ and chirp $C$ values. Lossless case.

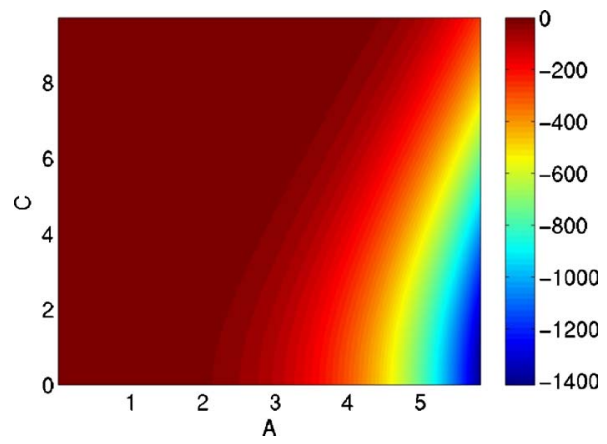

Fig. 3. (Color online) Density plot of the part of the Hamiltonian $\mathcal{H}_{\text {soliton}}$, see Eq. (8), transferred into the solitons generated from the input-chirped Gaussian profile, Eq. (9), as a function of the amplitude $A$ and chirp $C$ values. Lossless case. 
(a)
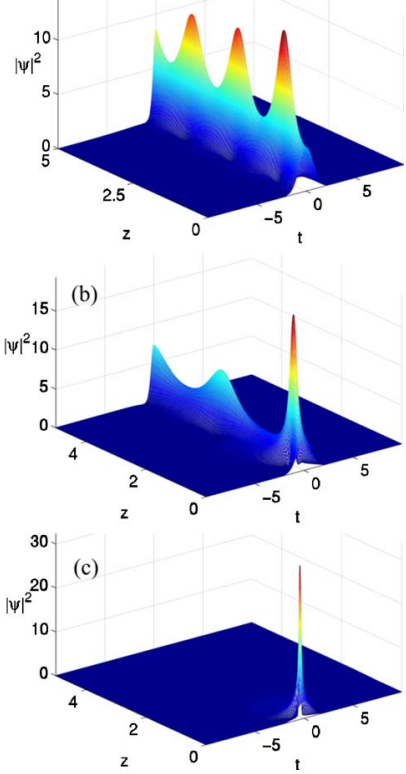

Fig. 4. (Color online) Propagation of a Gaussian pulse for different values of amplitude $A$ and chirp $C$ (see Fig. 1 for the corresponding number of bound states calculated for each case). Lossless case, $\alpha=0$ was assumed, (a) Here $A=2, C=0$, and a twosoliton bound state is formed, (b) $A=2, C=2$, and a single soliton is created, (c) For $A=2, C=7$, large chirp prevents the creation of solitons, and the power disperses quickly with the propagation.

chirped Gaussian pulses, Figs. 4(a)-4(c). For the nonlinear chirp (seeh-like phase function), there is the opposite trend in the behavior of the number of solitons versus chirp; also the initial pulse shape breaks into separating solitons and so-called Y-shaped ZSSP spectra occur. ${ }^{18,19,23}$ None of this is observed in our case of linear chirp.

\section{ZSSP MULTISOLITON DECOMPOSITION FOR THE SOLUTION OF LOSSY NLSE}

The results obtained so far concerned the quasi-lossless case $\alpha=0$, and for this situation, we explicitly demonstrated how the characteristics of the multisoliton bound state depend on the amplitude and chirp values of the input pulse. In this section, we consider how the fiber loss affects multisoliton bound states originating from chirped Gaussian input (9). When loss is small, $\alpha \ll 1$, the system is still close to the integrable case, and the adiabatic perturbation theory can be effectively applied to describe the evolution of the bound state. ${ }^{33}$ But when the loss is large enough (as in many situations of practical interest), the system can no longer be treated as almost integrable. Therefore qualitatively new physical effects not covered by the adiabatic perturbation theory can occur in this case. Figure 6 presents the evolution of the initial pulse Eq. (9) with $A=5, C=0$, over the distance $L=1$ (this distance corresponds to $25 \mathrm{~km}$ in real world units) with loss coefficient $\alpha=1.15(0.2 \mathrm{~dB} / \mathrm{km}$ in real world units $)$. The initial pulse corresponds to a five-soliton bound state in Fig. 1 [the associated ZSSP (4) possesses five welldistanced purely imaginary discrete eigenvalues]. The resulting complicated dynamics of the pulse is shown in Fig. 6 . It can be readily seen that the pulse, while decreasing in power, also breaks up into separate solitons or boundmultisoliton complexes, and each of these localized pulses decays individually. For two bound solitons, this effect was first reported in Ref. 34 where, however, splitting of the bound state was determined visually by the resulting pulse broadening (see also Ref. 35). We present here results of the spectral data ZSSP consideration that gives important new information concerning this effect. The ZSSP analysis performed at the fiber span output [i.e., the resulting output profile was taken as a potential of the ZSSP, Eq. (4)] confirms that there are still five discrete states present in the output pulse. While the imaginary parts of the eigenvalues (soliton amplitudes) unsurprisingly decrease due to the loss, another interesting effect is the appearance of nonzero real parts of the eigenvalues. In other words, the resulting multisoliton breather becomes unstable and breaks into soliton pairs, escaping symmetrically from the origin, while losing power due to loss. In Fig. 7, we present the results of the ZSSPdecomposition analysis for several sections taken at $z=0$, $z=0.13, z=0.54$, and $z=0.73$ for Figs. $7(\mathrm{a})-7(\mathrm{~d})$ correspondingly. We observed that the discrete eigenvalues of ZSSP evolve nonadiabatically, giving rise to instabilities.

The eigenvalues, initially located on the imaginary axis move closer to each other, and eventually collide and disperse, acquiring nonzero real parts (i.e., the individual solitons gain nonzero velocities). This corresponds to the breakup of the bound multisoliton state, which can be visually observed in Figs. 7. The detailed study of the bound-state instability and disintegration described

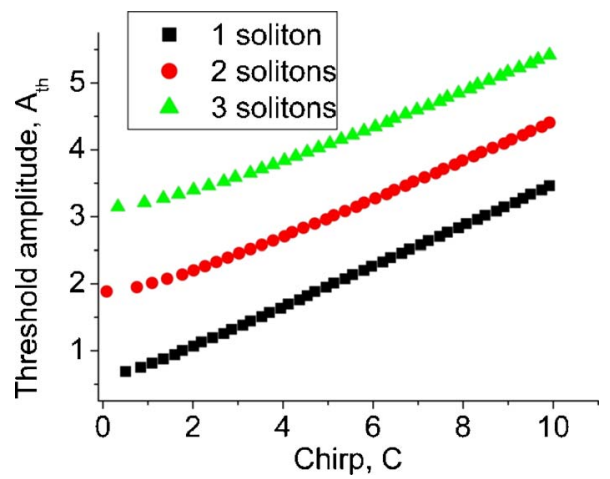

Fig. 5. (Color online) Amplitude threshold for soliton creation versus chirp. Lossless case.

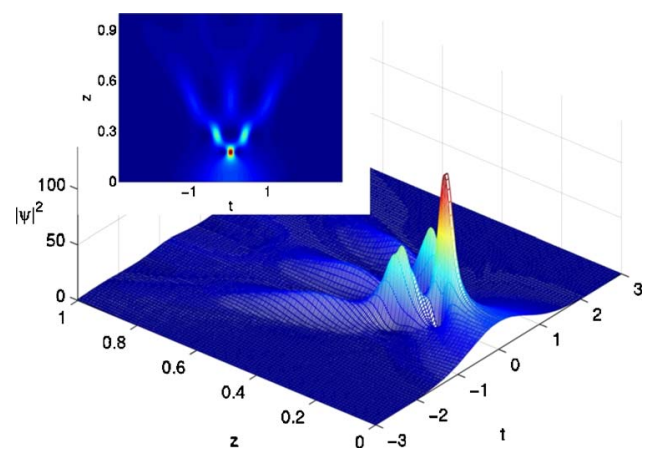

Fig. 6. (Color online) Breaking of a bound five-soliton state in a lossy fiber. The initial profile was given by Eq. (9) with $A=5, C$ $=0$. Pulse power is also shown as a density-plot in the inset. The propagation distance was $L=1$. 

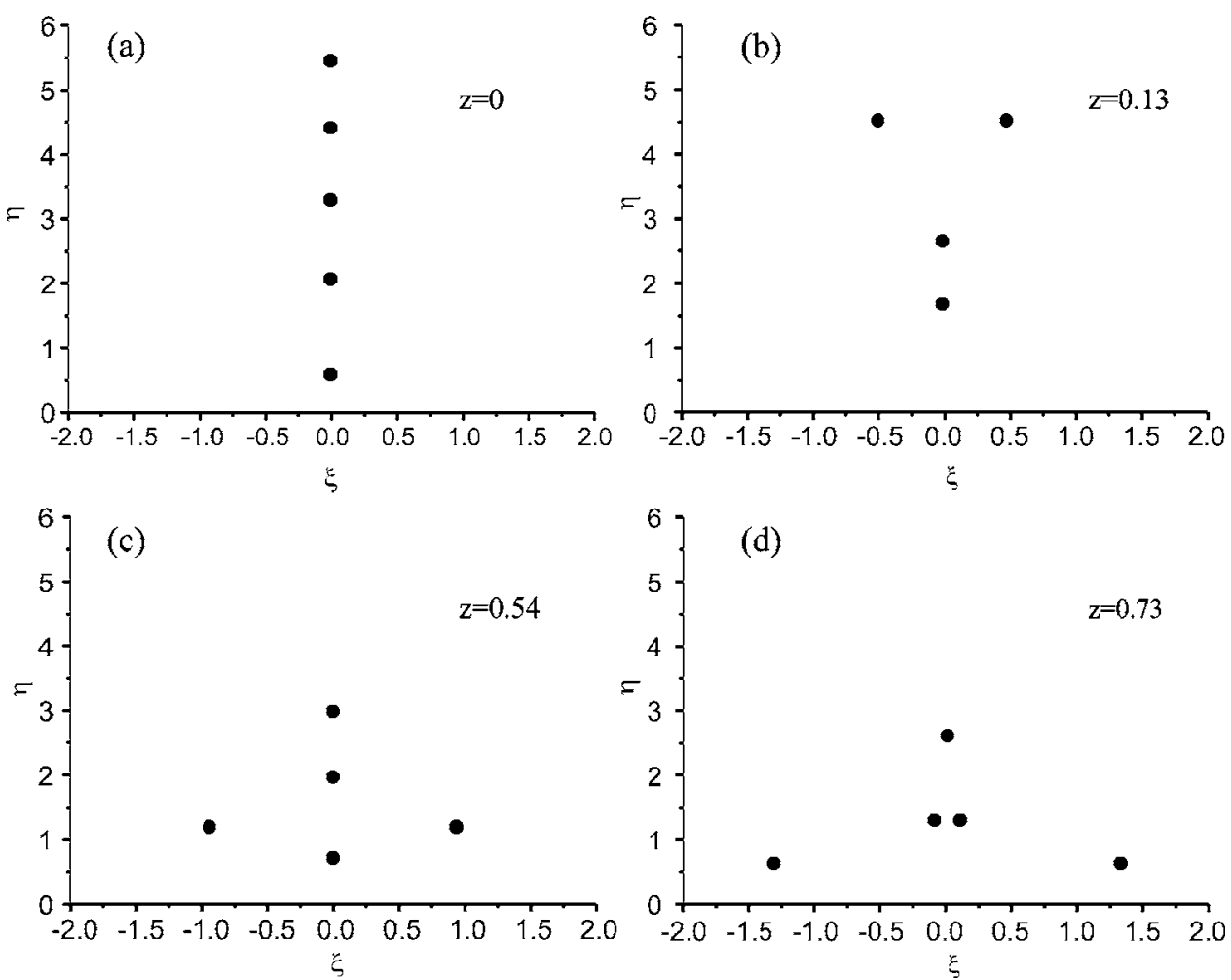

Fig. 7. Evolution of discrete ZSSP eigenvalues with the propagation distance $z$, corresponding to the lossy case shown in Fig. 6 and the same parameters of the initial chirped Gaussian pulse. (a) At $z=0$, we have five well-separated eigenvalues located at the imaginary axis, so that the bound state is the so-called breather state and soliton velocities are 0 . (b) At $z=0.13$, the lowest eigenvalue is now below the soliton threshold and also a pair of eigenvalues acquires symmetric real parts (velocities). (c) At $z=0.54$, the picture is qualitatively the same as in the case (b), but now the lowest eigenvalue reappears again. (d) At $z=0.73$, one observes a separation of yet another pair of eigenvalues.

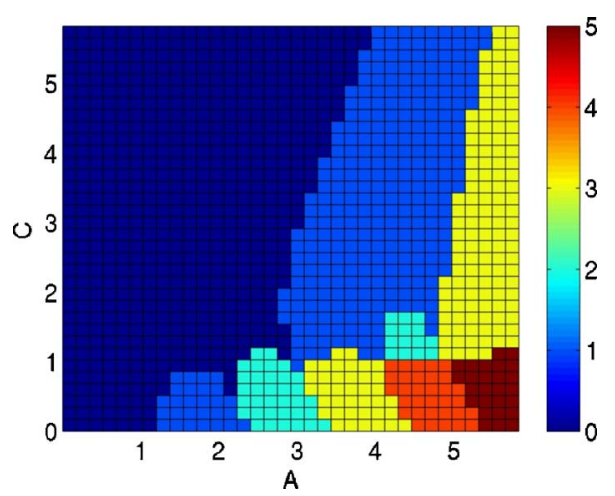

Fig. 8. (Color online) Density plot of the number of solitons [numbers of discrete eigenvalues for the associated ZSSP, Eq. (4)] generated from the input-chirped Gaussian profile, Eq. (9), as a function of the input amplitude $A$ and chirp $C$ values. Lossy case, $\alpha=1.15$, propagation distance $L=1$.

above is beyond the scope of this paper and will be published elsewhere.

Finally in Figs. 8-10, we plot the number of soliton states, relative power, and Hamiltonian for the lossy line, to be compared with Figs. 1-3, as functions of the input pulse parameters - the input amplitude and chirp. The presence of loss brings about a decrease of the number of bound-soliton states thus resulting in the complicated palette shown in Fig. 7. Because the initial configuration breaks into symmetric soliton pairs decaying simultaneously, the number of soliton states decreases with propagation more rapidly than one would expect in the

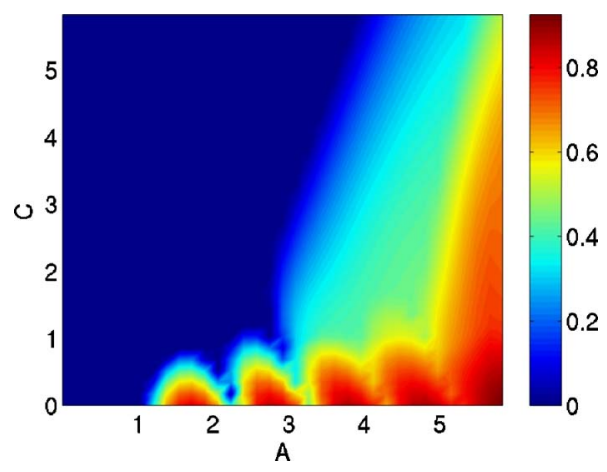

Fig. 9. (Color online) Density plot of the relative power of the solution, $\mathcal{N}_{\text {soliton }} /\left(\mathcal{N}_{\text {radiation }}+\mathcal{N}_{\text {soliton }}\right)$, generated from the inputchirped Gaussian profile, Eq. (9), as a function of the input amplitude $A$ and chirp $C$ values. Lossy case, $\alpha=1.15$, propagation distance $L=1$.

stable case-solitons annihilate both individually and in pairs. Also the output density plots of the relative power transferred to the solitons and of the $\mathcal{H}_{\text {soliton }}$ change correspondingly. The latter is the consequence of the complex waveform that occurs at the output of the lossy fiber span.

\section{CONCLUSION}

In the present paper, we have studied the process of pulse conversion from a linearly chirped Gaussian waveform into bound-multisoliton states. In the quasi-lossless regime, the resulting soliton eigenvalues are integrals of 


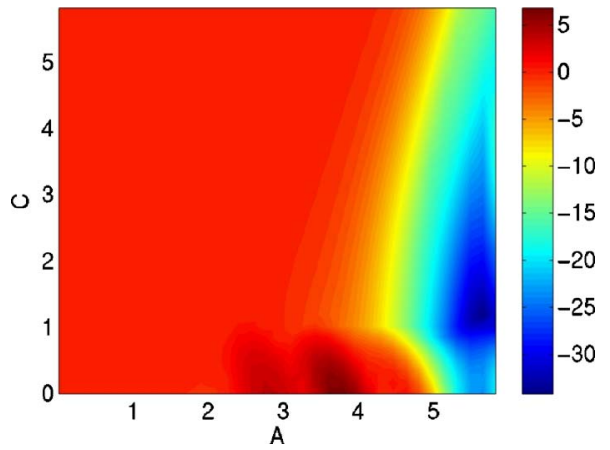

Fig. 10. (Color online) Density plot of the part of the Hamiltonian $\mathcal{H}$ transferred into the soliton part generated from the input-chirped Gaussian profile, Eq. (9), as a function of the input amplitude $A$ and chip $C$ values. Lossey case, $\alpha=1.15$, propagation distance $L=1$.

motion and are conserved during the signal evolution along the fiber while the linear radiation dissipates away. Therefore, the spectral data plots obtained for the input pulse can be used for the characterization of the output pulse properties. We have determined how the number of soliton states and the efficiency of conversion (defined as the relative power contained in the soliton solution) depend on both input pulse amplitude and chirp. In general, the increase of the initial pulse chirp reduces the number of bound-soliton states contained in the pulse. We have also obtained numerically the thresholds for the (multi)soliton-state formation as functions of the initial chirp parameter. In the lossy case, the overall power of the pulse decays exponentially with distance, and hence, the number of solitons decreases with the propagation as well. Also as predicted in Ref. 34, we observed a breakdown of the bound-multisoliton state by strong nonadiabatic loss. We must emphasize the difference between the soliton breakdown in the lossy case and a similar phenomenon in integrable lossless systems studied in Refs. $18,19,23$, and 24 . The lossless system is integrable; its eigenvalues are integrals of motion. Therefore the effect of the appearance of real parts of the eigenvalues is the intrinsic property of the incident pulse. When we treat the lossy case, however, the system becomes nonintegrable, and the eigenvalues are no longer integrals of motion. In particular, as occurs in our case, even when the ZSSP for the initial pulse does not contain real parts of the eigenvalues, such real parts may occur during the evolution. This effect is principally different from pulse instability in the lossless case and the two cases should not be confused. Applying the ZSSP formalism, we have described subsequent symmetric disintegration of the localized pulse into solitons, and multisoliton complexes contained a partial number of the initial bound states. We have quantitatively described the effectiveness of the pulse-to-soliton conversion in the lossy fiber lines with realistic parameters. We hope that these results can be useful for designing new photonic devices based on quasilossless fiber spans.

\section{ACKNOWLEDGMENTS}

The authors thank Keith Blow, Yuri Kivshar, Alexander Kovalev, and Michael Bogdan for helpful comments. The work was supported by EPSRC and the Royal Society.
The corresponding author, S. Derevyanko, can be reached via e-mail at sderevyanko@aston.ac.uk.

*On leave from the Institute for Radiophysics and Electronics, 61085 Kharkov, Ukraine.

\section{REFERENCES}

1. V. E. Zakharov and A. B. Shabat, "Exact theory of 2dimensional self-focusing and one-dimensional selfmodulations of waves in nonlinear media," Sov. Phys. JETP 34, 62-69 (1972).

2. A. Hasegawa and F. Tappert, "Transmission of stationary nonlinear optical pulses in dispersive dielectric fibres. I. Anomalous dispersion," Appl. Phys. Lett. 23, 142-144 (1973).

3. G. Agrawal, Nonlinear Fiber Optics (Academic, 2001).

4. A. Hasegawa and Y. Kodama, Solitons in Optical Communications (Clarendon, 1995).

5. E. Iannone, F. Matera, A. Mecozzi, and M. Settembre, Nonlinear Optical Communication Networks (Wiley, 1998).

6. L. F. Mollenauer and K. Smith, "Demonstration of soliton transmission over more than $4000-\mathrm{km}$ in fiber with loss periodically compensated by Raman gain," Opt. Lett. 13, 675-677 (1988).

7. L. F. Mollenauer, R. H. Stolen, and J. P. Gordon, "Experimental-observation of picosecond pulse narrowing and solitons in optical fibers," Phys. Rev. Lett. 45, 1095-1098 (1980).

8. B. Dany, P. Brindel, O. Leclerc, and E. Desurvire, "Transoceanic $440 \mathrm{Gbit} / \mathrm{s}$ system combining dispersionmanaged soliton transmission and new 'black-box' in-line optical regeneration," Electron. Lett. 35, 98-100 (1999).

9. B. Dany, P. Brindel, E. Pincemin, D. Rouvillain, and O. Leclerc, "Recovered efficiency of filter control in dispersionmanaged solitons for optical regeneration applications: analysis and experimental validation," Opt. Lett. 25, 793-795 (2000).

10. L. J. Wang, A. Agarwal, Y. K. Su, and P. Kumar, "All-optical picosecond-pulse packet buffer based on four-wave mixing loading and intracavity soliton control," IEEE J. Quantum Electron. 38, 614-619 (2002).

11. J. Ania-Castañón, "Quasi-lossless transmission using second-order Raman amplification and fibre Bragg grating," Opt. Express 12, 4372-4377 (2004).

12. J. D. Ania-Castañón and S. K. Turitsyn, "Analytical characterization of a quasi-lossless Raman-amplified fibre transmission scheme," in Lasers and Elctro-Optics Europe, Conference Digest for CLEO Europe 2005 (Optical Society of America, 2005), pp. CJ5-5-TUE.

13. J. D. Ania-Castañón, T. J. Ellingham, R. Ibbotson, X. Chen, L. Zhang, and S. K. Turitsyn, "Ultralong Raman fiber lasers as virtually lossless optical media," Phys. Rev. Lett. 96, 023902 (2006).

14. L. V. Hmurcik and D. J. Kaup, "Solitons created by chirped initial profiles in coherent pulse propagation," J. Opt. Soc. Am. 69, 597-604 (1979).

15. D. Burak and W. Nasalski, "Gaussian beam to spatial soliton formation in Kerr media,” Appl. Opt. 33, 6393-6401 (1994).

16. D. Burak, "Steering of bright-soliton pairs excited by symmetrical and real initial profiles," Phys. Rev. A 52, 4054-4058 (1995).

17. D. Krylov, L. Leng, K. Bergman, J. C. Bronski, and J. N. Kutz, "Observation of the breakup of a prechirped $N$-soliton in an optical fiber," Opt. Lett. 24, 1191-1193 (1999).

18. A. I. Maumistov and Y. M. Sklyarov, "Influence of the regular phase modulation on formation of optical solitons," Kvantovaya Elektron. (Kiev) 14, 796-803 (1987) [Sov. J. Quantum Electron. 17, 500-504 (1987)].

19. M. Desaux, L. Helczynski, D. Anderson, and M. Lisak, "Propagation properties of chirped soliton pulses in optical nonlinear Kerr media," Phys. Rev. E 65, 056602 (2002). 
20. K. J. Blow and D. Wood, "The evolution of solitons from non-transform limited pulses," Opt. Commun. 58, 349-354 (1986).

21. D. J. Kaup and B. A. Malomed, "Variational principle for the Zakharov-Shabat equations," Physica D 84, 319-328 (1995).

22. M. Desaix, D. Anderson, and M. Lisak, "Variational approach to the Zakharov-Shabat scattering problem," Phys. Rev. E 50, 2253-2256 (1994).

23. J. C. Bronski, "Semiclassical eigenvalue distribution of the Zakharov-Shabat eigenvalue problem," Physica D 97, 376-397 (1996)

24. J. C. Bronski and J. N. Kutz, "Numerical simulation of the semiclassical limits of the focusing nonlinear Schrodinger equation," Phys. Lett. A 254, 325-336 (1999).

25. M. Desaix, D. Anderson, M. Lisak, and M. L. QuirogaTeixeiro, "An approximation procedure for the Zakharov-Shabat scattering problem for real singlehumped potentials," J. Phys. A 29, 2493-2498 (1996).

26. S. Burtsev, R. Camassa, and I. Timofeyev, "Numerical algorithms for the direct spectral transform with applications to nonlinear Schrodinger type systems," J. Comput. Phys. 147, 166-186 (1998).

27. Y. S. Kivshar, "On the soliton generation in optical fibers," J. Phys. A 22, 337-340 (1988).

28. Y. S. Kivshar, Nonlinear Physics Centre, Research School of Physical Sciences and Engineering, The Australian National University, Canberra, Australian Capital Territory 0200, Australia (personal communication, 2006).

29. E. A. Kuznetsov, A. V. Mikhailov, and I. A. Shimokhin, "Nonlinear interaction of solitons and radiation," Physica D 87, 201-215 (1995).

30. W. L. Kath and N. F. Smyth, "Soliton evolution and radiation loss for the nonlinear Schrodinger equation," Phys. Rev. E 51, 1484-1492 (1995).

31. M. Böhm and F. Mitschke, "Soliton-radiation beat analysis," Phys. Rev. E 73, 066615 (2006).

32. J. Satsuma and N. Yajima, "Initial value problems of onedimensional self-modulation of nonlinear waves in dispersive media," Suppl. Prog. Theor. Phys. 55, 284-306 (1974).

33. Y. S. Kivshar and B. A. Malomed, "Dynamics of solitons in nearly integrable systems," Rev. Mod. Phys. 61, 763-915 (1989).

34. E. M. Dianov, Z. S. Nikonova, and V. N. Serkin, "The effect of optical losses on pulse propagation dynamics in singlemode fiber waveguides," Kvantovaya Elektron. (Kiev) 13 331-337 (1986) [Sov. J. Quantum Electron. 11, 219-222 (1986)].

35. K. J. Blow and N. J. Doran, "The asymptotic dispersion of soliton pulses in lossy fibers," Opt. Commun. 52, 367-370 (1985). 